\title{
Prescrição Antibiótica no Ambulatório em Doentes \\ Pediátricos com Patologia Respiratória
}

\section{Antibiotic Prescribing in Ambulatory Care of Pediatric Patients with Respiratory Infections}

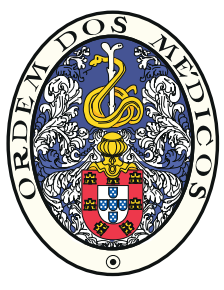

\author{
Joana Verdelho ANDRADE $\triangle^{1}$, Pedro VASCONCELOS ${ }^{2}$, Joana CAMPOS ${ }^{1}$, Teresa CAMURÇA ${ }^{2}$
}

Acta Med Port 2019 Feb;32(2):101-110 - https://doi.org/10.20344/amp.11111

\section{RESUMO}

Introdução: As infeções do trato respiratório são das patologias que mais frequentemente motivam a prescrição antibiótica em idade pediátrica. Os objetivos deste estudo foram identificar a frequência e padrão de prescrição de antibacterianos na patologia respiratória. Material e Métodos: Durante dois anos (divididos pela apresentação das normas de orientação clínica), os dados foram obtidos através da consulta de processos clínicos de crianças com patologia respiratória. Utilizaram-se os testes qui-quadrado ou teste exato de Fisher para testar associações entre variáveis, assumindo-se significado estatístico quando $p<0,05$.

Resultados: Realizaram-se 547 consultas (idade média de seis anos $\pm 5,3,55 \%$ do género masculino). A pesquisa do antigénio do Streptococcus do grupo A na orofaringe foi realizada mais frequentemente por internos de Pediatria $(p=0,005)$. A radiografia de tórax foi mais pedida pelo especialista de Medicina Geral e Familiar $(p=0,033)$. Prescreveu-se antibiótico em $87 \%$ pneumonias, $84 \%$ otites médias agudas, $68 \%$ amigdalites, $25 \%$ laringites, $17 \%$ infeções respiratórias superiores e $16 \%$ bronquiolites agudas. 0 especialista de Medicina Geral e Familiar prescreveu mais antibiótico que o interno de Pediatria na amigdalite aguda $(p=0,003)$ e na otite média aguda $(p=0,013)$. O antibiótico mais prescrito foi amoxicilina (61\%). Não houve diferenças entre os dois períodos estudados quanto ao número de prescrições e escolha de antibiótico das patologias estudadas.

Discussão: A prescrição de antibióticos foi elevada e numa proporção significativa a antibioterapia poderia ser ajustada. Realça-se que não houve diferenças na prescrição após a apresentação das normas de orientação clínica.

Conclusão: Considera-se necessária uma melhoria da prescrição antibiótica pediátrica no ambulatório.

Palavras-chave: Antibacterianos; Assistência Ambulatorial; Criança; Infecções Respiratórias; Prescrição Inadequada; Utilização de Medicamentos

\section{ABSTRACT}

Introduction: Respiratory tract infections represent the most frequent conditions in pediatric clinical practice that motivate antibiotic prescribing. The objective was to identify the frequency and pattern of antibacterial prescribing in respiratory diseases.

Material and Methods: Over a period of two years (divided by the presentation of the clinical guideline standards) data was collected from clinical records of children with respiratory disease. Chi-square tests or Fisher's exact test were used to test associations between variables, statistical significance $p<0.05$

Results: There were 547 visits (mean age 6 years $\pm 5.3,55 \%$ male gender). Analysis for Group A Streptococcus of the oropharynx was most frequently requested by pediatric residents $(p=0.005)$. Chest $\mathrm{x}$-rays were more frequently requested by the Family Physician $(p$ $=0.033$ ). An antibiotic was prescribed in $87 \%$ of pneumonias, $84 \%$ acute otitis media, $68 \%$ acute tonsillitis, $25 \%$ laryngitis, $17 \%$ upper respiratory infections, $16 \%$ acute bronchiolitis. The Family Physician prescribed antibiotics more often than the Pediatrics resident in acute tonsillitis $(p=0.003)$ and in acute otitis media $(p=0.013)$. The most frequently prescribed antibiotic was amoxicillin $(61 \%)$. There were no significant differences between the two periods studied regarding the number of prescriptions and antibiotic choice of the conditions studied.

Discussion: Antibiotic prescribing in pediatric acute respiratory infections was high and the choice of antibiotic therapy could be adjusted. We found no difference in antibiotic prescribing after the presentation of the clinical guideline standards.

Conclusion: An improvement in the antibiotic prescription in children and adolescents in the outpatient clinic is considered necessary. Keywords: Ambulatory Care; Anti-Bacterial Agents; Child; Drug Utilization; Inappropriate Prescribing; Respiratory Tract Infections

\section{INTRODUÇÃO}

As infeções do trato respiratório representam uma das patologias que mais frequentemente motivam a prescrição antibiótica em idade pediátrica. $\mathrm{O}$ uso racional de medicamentos é fundamental na prática médica com especial relevância no caso dos antibióticos. A sua utilização inadequada pode afectar a qualidade assistencial e aumentar os custos em saúde, assim como favorecer o aparecimento de efeitos secundários e resistências bacterianas. ${ }^{1-10} \mathrm{Na}$ prática clínica em geral e em particular em pediatria, têm surgido preocupações referentes à dificuldade crescente no tratamento de infeções comuns, facto intimamente relacio- nado com o uso excessivo destes fármacos, sendo necessário manter uma avaliação contínua da sua prescrição. ${ }^{11-19}$

A nível internacional, constatou-se uma grande prevalência do uso de antibióticos, especialmente em doentes em idade pré-escolar, com uma taxa de 2,2 prescrições por criança ao ano e uma estimativa de utilização injustificada em pelo menos um terço dessas prescrições. ${ }^{20,21}$ Entre os quadros infeciosos que afetam a população infantil destaca-se a do foro respiratório, que têm na sua maioria uma etiologia vírica e constituem, portanto, um grupo importante de prescrição potencialmente errónea. ${ }^{22}$ Um estudo

\footnotetext{
1. Serviço Pediatria. Centro Hospitalar Tondela-Viseu. Viseu. Portugal.

2. Departamento de Medicina Geral e Familiar. Unidade de Saúde Familiar Viseu-Cidade. Agrupamento Centros de Saúde Dão Lafões. Viseu. Portugal.

$\triangle$ Autor correspondente: Joana Verdelho Andrade. joanaverdelhoandrade@gmail.com

Recebido: 23 de julho de 2018 - Aceite: 22 de outubro de 2018 | Copyright $\odot$ Ordem dos Médicos 2019
} 
espanhol, realizado em onze serviços de urgência pediátrica revelou um uso inadequado de antibióticos em infeções respiratórias entre $14 \%$ a $80 \%$ dos casos. $^{23}$

O aumento das resistências aos antibióticos é um problema grave de saúde pública que em grande parte dos casos resulta da utilização indiscriminada e errada dos antibióticos. Em resposta a este problema, surgiram iniciativas tanto locais, como nacionais e internacionais para implementar programas de melhoria da qualidade da prescrição, não só com medidas educacionais aos profissionais de saúde e à população em geral, mas também com a introdução de protocolos ou guias de tratamento tanto intra como extra-hospitalares. ${ }^{26-31} \mathrm{O}$ uso racional dos antibióticos constitui aliás um dos parâmetros valorizados para determinar a qualidade dos cuidados médicos, pelo que o seu uso incorreto indicaria uma menor qualidade assistencial.

Em Portugal, há Normas de Orientação Clínica (NOC) da Direção-Geral da Saúde, bem como guias clínicos e documentos de consensos que reconhecem a evidência científica para o uso de antibióticos em idade pediátrica. ${ }^{26-31}$ No entanto, desconhece-se se a sua utilização é racional ou se os médicos das áreas de Pediatria $(P)$ e Medicina Geral e Familiar (MGF) reconhecem estes guias no momento da prescrição antibiótica. Num cenário ideal, a prescrição não hospitalar seria cerca de dez doses diárias por mil habitantes por dia enquanto a nível hospitalar cerca de três. ${ }^{32-36} \mathrm{O}$ consumo global de antibacterianos em Portugal nos Cuidados de Saúde Primários mantém-se num nível ainda elevado (21,6 doses diárias por mil habitantes por dia), apesar de abaixo da média da Europa $(21,9)$, sendo as penicilinas e o grupo de macrólidos, lincosamidas e estreptograminas mais frequentemente utilizados. ${ }^{34}$ Apesar da relevância do tema, desconhecem-se os padrões de prescrição em Pediatria nos serviços hospitalares e no ambulatório em Portugal, particularmente o seu impacto nos quadros infecciosos respiratórios. É necessária a obtenção desses dados para se optimizar a utilização dos recursos, assim como a realização de um seguimento dos processos assistenciais. ${ }^{22}$

Muitos países reduziram de forma bem-sucedida a prescrição antibiótica no ambulatório, sendo que ainda é a este nível que a maioria dos antibióticos são prescritos. ${ }^{9}$ No entanto, há vários constrangimentos para a redução efetiva da prescrição inapropriada dos antibióticos, onde se incluem as expectativas do doente e profissional de saúde,$^{37}$ a escassa sensibilização do doente para a existência de resistências ${ }^{38}$ e a percepção dos clínicos dos cuidados de saúde primários de que o risco de resistência aos antimicrobianos é mínimo. ${ }^{39,40}$ De qualquer forma, não é só a prescrição médica que determina o consumo de antibióticos pelas crianças, uma vez que muitas vezes são os próprios cuidadores que a iniciam, o que acontece tanto mais frequentemente quanto menor o nível sociocultural da família. ${ }^{40,41}$

Os objetivos deste estudo foram conhecer a percentagem de consultas por quadros infeciosos respiratórios, os diagnósticos mais frequentes, identificar a frequência de prescrição de antibacterianos nas infeções respiratórias, apreciar o padrão dessa utilização nos Cuidados de Saúde Primários e a adequação da prescrição antibiótica às recomendações internacionais, e comparar estes parâmetros prévios e posteriores à apresentação de NOC de diagnóstico e tratamento de otite média aguda, amigdalite aguda, bronquiolite aguda e pneumonia adquirida na comunidade da Direção-Geral da Saúde.

\section{MATERIAL E MÉTODOS \\ População acessível}

Estudou-se a abordagem diagnóstica e tratamento de infeções respiratórias de utentes em idade pediátrica (inferior a 18 anos) inscritos numa Unidade de Saúde, com os seguintes diagnósticos, segundo a Classificação Internacional de Assistência Primária (ICPC-2) dos cuidados de saúde primários: otite média aguda ( $\mathrm{H} 71)$; infeção estreptocócica da orofaringe (R72); infeção aguda do aparelho respiratório superior (R74); amigdalite aguda (R76); laringite (R77); bronquite /bronquiolite aguda (R78); gripe (R80); pneumonia (R81); infeção respiratória, outra (R83); asma (R96); doença respiratória, outra (R99); através da consulta do processo clínico informatizado (SClínico ${ }^{\circledR}$ ) e em caso de necessidade do contacto telefónico com utente ou tutor legal.

Excluiu-se os casos em que os registos de consulta estavam incompletamente preenchidos para qualquer uma das variáveis estudadas.

\section{Período do estudo}

Duração total do estudo de dois anos através da avaliação dos registos clínicos durante um ano a iniciar a 16 de outubro de 2014 (primeira avaliação - retrospetiva) e segundo período com igual duração a iniciar a 16 de outubro de 2015 (segunda avaliação - prospetiva).

\section{Desenho do estudo}

Realizou-se uma avaliação interna, ambispetiva, interpares e de base institucional com intervenção educacional focada no profissional. Realizou-se uma apresentação formal e sistemática de NOC da Direção-Geral da Saúde de "Diagnóstico e Tratamento da Otite Média Aguda em Idade Pediátrica, Diagnóstico e Tratamento da Amigdalite Aguda em Idade Pediátrica, Diagnóstico e Tratamento da Pneumonia Adquirida na Comunidade em Idade Pediátrica, Diagnóstico e Tratamento da Bronquiolite Aguda na Idade Pediátrica" a 16 de outubro de 2015. O estudo foi conduzido durante um período de dois anos (divididos equitativamente pela apresentação das NOC). Avaliou-se a proporção de episódios de cada diagnóstico, abordagem e tratamento de acordo com o padrão e tipo de prescrição. As variáveis estudadas em relação aos utentes pediátricos foram a idade, o género, antecedentes perinatais, antecedentes patológicos, alergias medicamentosas, cumprimento do programa nacional de vacinação e cobertura com vacinas extra-programa e utilização de antibioterapia prévia nos últimos doze meses. Relativamente à abordagem 
diagnóstica e terapêutica analisou-se que médico realizou a consulta, quanto ao grau e especialidade médica (especialista ou interno de MGF e interno de P), se foi solicitado algum exame complementar de diagnóstico e se foi prescrito antibiótico (grupo e duração de tratamento). Dividiu-se a amostra segundo a especialidade (MGF e P).

\section{Análise estatística}

Os dados foram recolhidos e codificados numa tabela inserida numa aplicação informática $\mathrm{Excel}^{\circledR}$, versão 2011
(Microsoft Corporation, EUA) para tratamento estatístico, complementado com software SPSS ${ }^{\circledR}$, versão 20 para Mac (SPSS, IL, EUA). O tratamento dos dados teve uma componente descritiva com distribuição de frequências, bem como analítica. Foi utilizado o teste exato de Fisher e qui-quadrado de independência. Considerou-se significância estatística quando o valor $-p$ dos testes for $p<0,05$.

\section{Considerações éticas}

O estudo foi aprovado pela Comissão de Ética para a

Tabela 1 - Proporção de consultas realizadas segundo o tipo de médico e patologia

\begin{tabular}{|c|c|c|c|c|}
\hline Consulta realizada por: & $\begin{array}{c}\text { Especialista de MGF } \\
\mathrm{n}(\%)\end{array}$ & $\begin{array}{c}\text { Interno de Pediatria } \\
\mathrm{n}(\%)\end{array}$ & $\begin{array}{c}\text { Interno de MGF } \\
\mathrm{n}(\%)\end{array}$ & $\begin{array}{l}\text { Total } \\
\mathrm{n}(\%)\end{array}$ \\
\hline $\begin{array}{l}\text { Infeção aguda do aparelho } \\
\text { respiratório superior inespecífica }\end{array}$ & $88(43 \%)$ & $85(41 \%)$ & $33(16 \%)$ & $206(38 \%)$ \\
\hline Amigdalite aguda & $62(53 \%)$ & $36(31 \%)$ & $18(16 \%)$ & $116(21 \%)$ \\
\hline Otite média aguda & $53(59 \%)$ & $23(26 \%)$ & $13(15 \%)$ & $89(17 \%)$ \\
\hline Bronquiolite aguda & $25(59 \%)$ & $11(25 \%)$ & $6(16 \%)$ & $42(8 \%)$ \\
\hline Pneumonia & $22(57 \%)$ & $13(33 \%)$ & $3(10 \%)$ & $38(6 \%)$ \\
\hline Asma & $21(70 \%)$ & $2(5 \%)$ & $7(25 \%)$ & $30(5 \%)$ \\
\hline Gripe & $12(77 \%)$ & $1(6 \%)$ & $3(17 \%)$ & $16(3 \%)$ \\
\hline Laringite & $4(40 \%)$ & $5(50 \%)$ & $1(10 \%)$ & $10(2 \%)$ \\
\hline Total & $284(52 \%)$ & $175(32 \%)$ & $88(16 \%)$ & $547(100 \%)$ \\
\hline
\end{tabular}

MGF: medicina geral e familiar

Tabela 2 - Caraterização da amostra dos utentes pediátricos $(n=395)$

\begin{tabular}{|c|c|c|}
\hline \multicolumn{2}{|c|}{ Caraterização da amostra dos utentes pediátricos } & \multirow{2}{*}{$\begin{array}{c}\text { n (\%) } \\
155(45 \%)\end{array}$} \\
\hline Género & Feminino & \\
\hline & Masculino & $240(55 \%)$ \\
\hline \multirow[t]{6}{*}{ Idade } & Média & 6,1 anos $\pm 5,3$ \\
\hline & Mínimo, Máximo & 21 dias, 17 anos \\
\hline & $<1$ ano & $32(8 \%)$ \\
\hline & $1-5$ anos & $182(46 \%)$ \\
\hline & $6-9$ anos & $79(20 \%)$ \\
\hline & $>10$ anos & $102(26 \%)$ \\
\hline \multirow[t]{3}{*}{ Gravidez } & Idade gestacional & $367(93 \%)$ Termo \\
\hline & & 28 (7\%) Pré-termo \\
\hline & Vigiada (pelo menos 6 consultas) & $393(99,5 \%) \operatorname{sim}, 2(0,5 \%)$ não \\
\hline \multirow[t]{5}{*}{ Comorbilidades atuais } & $\mathrm{n}=207$ & \\
\hline & Excesso de peso ou obesidade & $130(33 \%)$ \\
\hline & Asma ou sibilância recorrente & $36(9 \%)$ \\
\hline & Problemas de neurodesenvolvimento & $12(3 \%)$ \\
\hline & Outra & $12(3 \%)$ \\
\hline \multirow[t]{5}{*}{ Vacinação } & Programa Nacional de Vacinação atualizado & $391(99 \%)$ \\
\hline & Vacina antipneumocócica & $300(76 \%)$ \\
\hline & Vacina antirotavírus & $122(31 \%)$ \\
\hline & Vacina anti-hepatite A & $95(24 \%)$ \\
\hline & Vacina antimeningococo B & $87(22 \%)$ \\
\hline Alergias medicamentosas & Sim & $\begin{array}{c}2(0,4 \%) \\
\text { Amoxicilina e ibuprofeno }\end{array}$ \\
\hline \multicolumn{2}{|c|}{ Antibioterapia nos últimos doze meses } & $47(12 \%) \operatorname{sim}, 348$ (88\%) não \\
\hline
\end{tabular}


Tabela 3 - Caraterização da proporção de casos por diagnóstico a qual foi pedido algum tipo de exame complementar de diagnóstico e que receberam prescrição antibiótica por grupo de especialidade

\begin{tabular}{|c|c|c|c|c|}
\hline & $\begin{array}{c}\text { Grupo de MGF } \\
n(\%)\end{array}$ & $\begin{array}{c}\text { Grupo de Pediatria } \\
\mathrm{n}(\%)\end{array}$ & $p$ & $\begin{array}{l}\text { Total } \\
\mathrm{n}(\%)\end{array}$ \\
\hline \multicolumn{5}{|c|}{ Realização de exames complementares de diagnóstico } \\
\hline Totalidade das consultas & $34(9 \%)$ & $15(9 \%)$ & & $49(9 \%)$ \\
\hline Amigdalite aguda & $10(13 \%)$ & $14(39 \%)$ & $<0,001^{\#}$ & $24(21 \%)$ \\
\hline Pneumonia & $15(60 \%)$ & $1(8 \%)$ & $0,009^{\#}$ & $16(42 \%)$ \\
\hline Infeção respiratória superior inespecífica & $4(3 \%)$ & $0(0 \%)$ & $0,273^{*}$ & $4(2 \%)$ \\
\hline Bronquiolite aguda & $3(10 \%)$ & $0(0 \%)$ & $0,956^{*}$ & $3(7 \%)$ \\
\hline Otite média aguda & $1(2 \%)$ & $0(0 \%)$ & $0,998^{*}$ & $1(1 \%)$ \\
\hline Exacerbação de asma & $1(4 \%)$ & $0(0 \%)$ & $0,950^{*}$ & $1(3 \%)$ \\
\hline Laringite aguda & $0(0 \%)$ & $0(0 \%)$ & - & $0(0 \%)$ \\
\hline Gripe & $0(0 \%)$ & $0(0 \%)$ & - & $0(0 \%)$ \\
\hline \multicolumn{5}{|l|}{ Prescrição antibiótica } \\
\hline Totalidade das consultas & $179(48 \%)$ & $52(30 \%)$ & & $231(42 \%)$ \\
\hline Amigdalite aguda & $62(78 \%)$ & $17(47 \%)$ & $0,001^{\#}$ & $79(68 \%)$ \\
\hline Otite média aguda & $59(89 \%)$ & $15(65 \%)$ & $0,013^{\#}$ & $74(83 \%)$ \\
\hline Infeção respiratória superior inespecífica & $28(23 \%)$ & $7(8 \%)$ & $0,011^{\#}$ & $35(17 \%)$ \\
\hline Pneumonia & $20(80 \%)$ & $13(100 \%)$ & $0,129^{\#}$ & $33(87 \%)$ \\
\hline Bronquiolite aguda & $7(23 \%)$ & $0(0 \%)$ & $0,296^{*}$ & $7(17 \%)$ \\
\hline Laringite aguda & $3(60 \%)$ & $0(0 \%)$ & $0,429^{*}$ & $3(30 \%)$ \\
\hline Gripe & $0(0 \%)$ & $0(0 \%)$ & - & $0(0 \%)$ \\
\hline Exacerbação de asma & $0(0 \%)$ & $0(0 \%)$ & - & $0(0 \%)$ \\
\hline
\end{tabular}

*: teste exato de Fisher; \#: teste qui-quadrado de independência; MGF: medicina geral e familiar

Saúde do Centro Hospitalar Tondela Viseu.

\section{RESULTADOS}

As consultas de agudos de utentes com menos de 18 anos neste período foram 1578 e registaram-se 547 consultas por patologia respiratória (35\%). Os diagnósticos mais frequentes foram a infeção aguda do aparelho respiratório superior inespecífica (38\%), a amigdalite aguda $(21 \%)$ e a otite média aguda (17\%) e as consultas foram realizadas com maior frequência por especialista de MGF (52\%) e interno de $\mathrm{P}(32 \%)$ (Tabela 1).

Observaram-se 395 utentes pediátricos neste período de um total de 6880 utentes inscritos com menos de 18 anos $(6 \%), 38 \%$ dos quais foram observados pelo menos mais de uma vez por episódio diferente do foro respiratório. Constatou-se uma ligeira predominância do género masculino (55\%) e uma idade média de 6 anos $\pm 5,3$ (mínimo 21 dias, máximo 17 anos). A patologia associada mais frequente foi o excesso de peso ou obesidade (33\%). A maio- ria dos doentes (99\%) tinha o programa nacional de vacinação atualizado e $76 \%$ a vacina antipneumocócica (Tabela 2).

Prescreveu-se antibiótico mais frequentemente nas pneumonias, seguido da prescrição para otites médias agudas e amigdalites agudas (Tabela 3). A escolha mais preponderante recaiu na amoxicilina, seguido da amoxicilina e ácido clavulânico e de macrólido (Tabela 4, Fig. 1). A proporção de episódios tratados em crianças menores do que dois anos foi inferior em todos os diagnósticos, excepto para o diagnóstico de otite média aguda, em que $95 \%$ dos episódios receberam antibiótico. A proporção de amigdalites agudas tratadas com antibiótico foi maior nas crianças mais velhas, atingindo $88 \%$ nos adolescentes com mais de 10 anos de idade (Fig. 2).

\section{Amigdalite aguda}

Realizou-se pesquisa do Streptococcus do grupo A na orofaringe em $21 \%$ das amigdalites agudas, mais frequentemente requisitado pelo grupo $\mathrm{P}$ do que pelo grupo de

Tabela 4 - Caraterização da escolha de antibioterapia prescrita $(n=231)$

\begin{tabular}{|c|c|c|c|c|}
\hline Prescrição antibiótica & $\begin{array}{c}\text { Grupo de MGF } \\
n(\%)\end{array}$ & $\begin{array}{c}\text { Grupo de Pediatria } \\
n(\%)\end{array}$ & $\boldsymbol{p}^{\#}$ & $\begin{array}{l}\text { Total } \\
\mathrm{n}(\%)\end{array}$ \\
\hline Amoxicilina & $102(57 \%)$ & $35(68 \%)$ & 0,059 & $137(59 \%)$ \\
\hline Amoxicilina e Ácido Clavulânico & $39(22 \%)$ & $7(13 \%)$ & 0,154 & $46(20 \%)$ \\
\hline Macrólido & $25(14 \%)$ & $9(17 \%)$ & 0,554 & $34(15 \%)$ \\
\hline Cefalosporina $2^{\mathrm{a}} / 3^{\mathrm{a}}$ geração & $13(7 \%)$ & $1(2 \%)$ & 0,226 & $14(6 \%)$ \\
\hline
\end{tabular}

\#: teste qui-quadrado de independência; MGF: medicina geral e familiar 


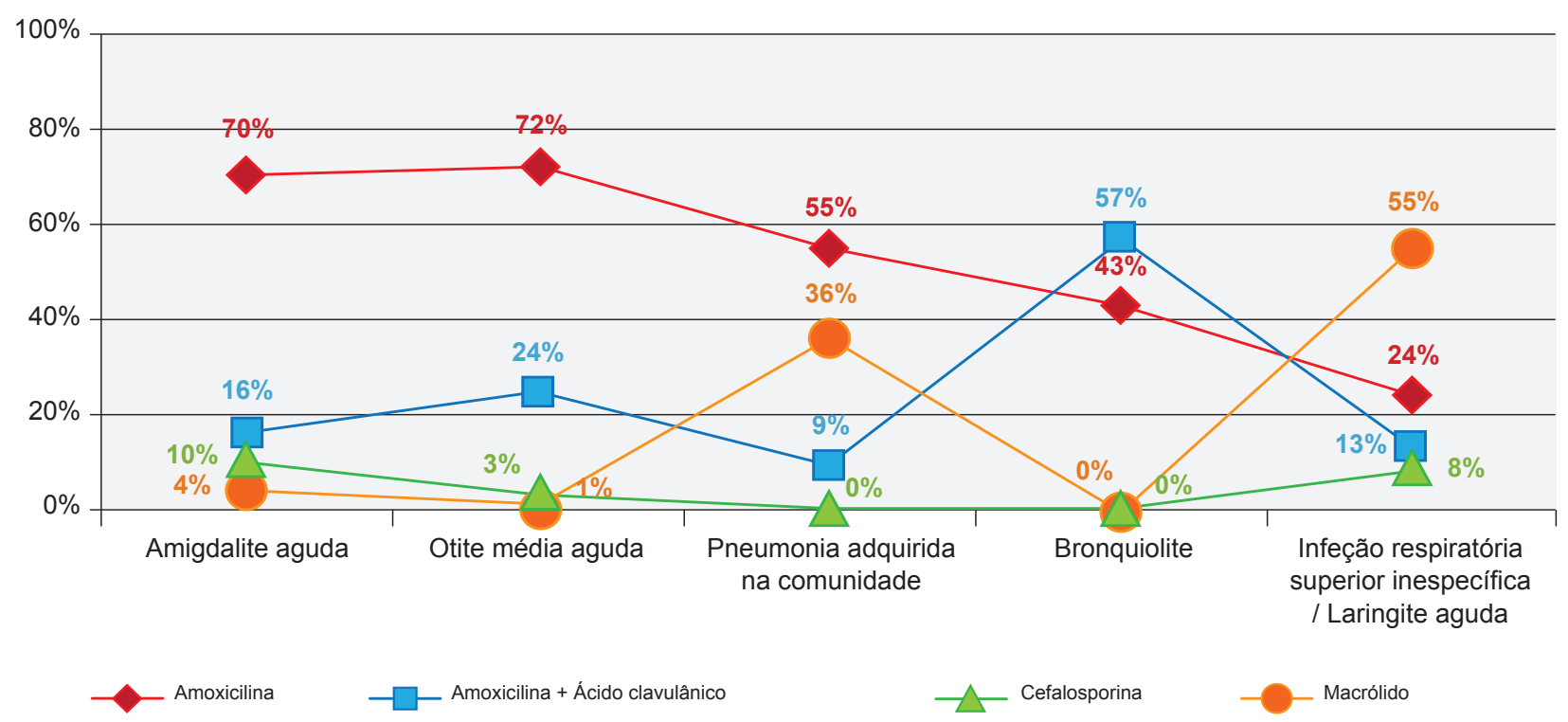

Figura 1 - Padrão de prescrição antibiótica nos episódios tratados

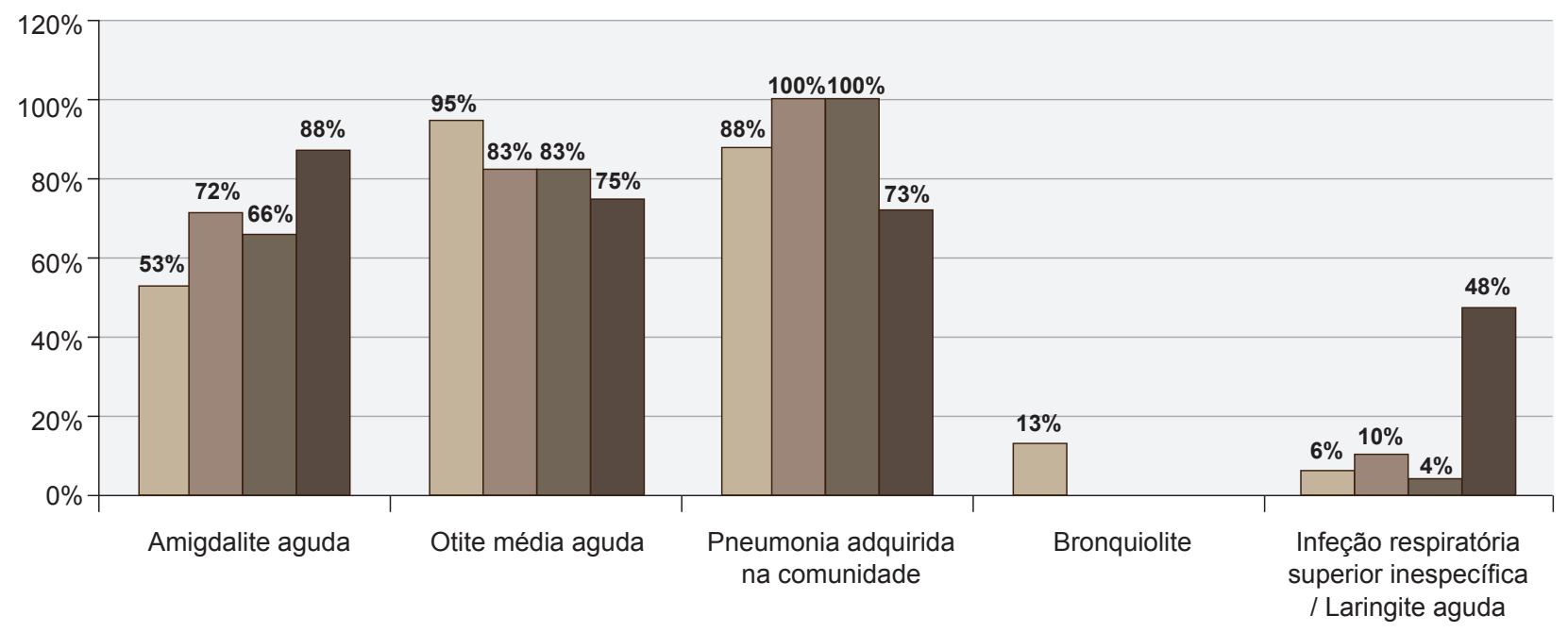

$\square<2$ anos $\square 2-5$ anos $\square 6-9$ anos $\square>10$ anos

Figura 2 - Proporção de episódios que recebem prescrição antibiótica, por tipo de diagnóstico e grupo de idade

MGF $(p<0,001)$ (Tabela 3).

A prescrição antibiótica foi realizada em 79 casos de amigdalite aguda (68\%). A escolha antibiótica foi a amoxicilina $(n=55 ; 70 \%)$, amoxicilina e ácido clavulânico $(n=$ $13 ; 16 \%)$, cefalosporina de segunda geração $(n=8 ; 10 \%)$ e macrólido $(n=3 ; 4 \%)$. O grupo de MGF prescreveu mais frequentemente antibiótico $(p=0,001)$ (Tabela 3$)$ e ambos os grupos prescreveram antibioterapia mais frequentemente nos doentes com idade igual ou superior a dois anos ( $p$ $=0,034$ ) (Tabela 5). Os profissionais de saúde cumpriram a NOC frequentemente na escolha da amoxicilina (sem diferença estatisticamente significativa entre os grupos), no entanto, os internos de Pediatria optavam mais frequente pelos dez dias aconselhados na NOC $(p=0,002)$ (Tabela $5)$.

\section{Otite média aguda}

$O$ grupo de MGF prescrevia mais frequentemente antibiótico do que o interno de $\mathrm{P}$ na otite média aguda $(p=$ 0,013 ) (Tabela 3 ), que cumpriu a NOC na atitude expectante na otite média aguda $(p=0,046)$ (Tabela 5).

A prescrição antibiótica foi realizada em 74 casos (83\%). Os profissionais de saúde cumpriram a NOC na maioria dos casos quanto à escolha da antibioterapia: amoxicilina $(n=53 ; 72 \%)$, amoxicilina e ácido clavulânico $(n=18$; $24 \%$ ), cefalosporina de segunda ou terceira geração ( $n=$ 2; $3 \%$ ) e macrólido ( $n=1 ; 1 \%)$. Quando a escolha recaiu num beta-lactâmico $(n=55 ; 74 \%), 87 \%(n=48)$ optou por prescrever durante sete a dez dias, mesmo nos casos com indicação para tratamento mais curto $(n=12 ; 25 \%)$. Dentro dos casos medicados com antibioterapia, $2 \%(n=2)$ foram reavaliados novamente em 48 - 72 horas por persistência 
Tabela 5 - Caraterização da escolha de antibioterapia prescrita na amigdalite aguda, otite média aguda, pneumonia adquirida na comunidade e bronquiolite aguda [ $1^{\mathrm{a}}$ parte]

\begin{tabular}{|c|c|c|c|c|}
\hline \multicolumn{5}{|l|}{ AMIGDALITE AGUDA } \\
\hline & & $\begin{array}{c}\text { Idade }<2 \text { anos } \\
n=19 \\
n(\%)\end{array}$ & $\begin{array}{c}\text { Idade } \geq 2 \text { anos } \\
n=97 \\
n(\%)\end{array}$ & $p$ \\
\hline \multirow[t]{3}{*}{ Prescrição antibiótica } & Sim & $9(47 \%)$ & $70(72 \%)$ & $\mathbf{0 , 0 3 4 ^ { \# }}$ \\
\hline & Não & $10(53 \%)$ & $27(28 \%)$ & \\
\hline & & $\begin{array}{c}\text { Grupo de MGF } \\
n=62 \\
n(\%)\end{array}$ & $\begin{array}{c}\text { Grupo de Pediatria } \\
n=17 \\
n(\%)\end{array}$ & $p$ \\
\hline \multirow[t]{2}{*}{ Prescrição antibiótica de acordo com NOC } & $\operatorname{Sim}$ & $42(68 \%)$ & $13(76 \%)$ & $0,465^{\#}$ \\
\hline & Não & $20(32 \%)$ & $4(24 \%)$ & \\
\hline \multirow[t]{3}{*}{ Duração antibiótica } & $7-8$ dias & $48(77 \%)$ & $6(35 \%)$ & $0,002^{\#}$ \\
\hline & 10 dias & $14(23 \%)$ & $11(65 \%)$ & \\
\hline & & $\begin{array}{c}1^{\circ} \text { ano } \\
\mathrm{n}=49 \\
\mathrm{n}(\%)\end{array}$ & $\begin{array}{c}2^{\circ} \text { ano } \\
\mathrm{n}=67 \\
\mathrm{n}(\%)\end{array}$ & p \\
\hline \multirow[t]{2}{*}{ Realização de exames complementares de diagnóstico } & $\operatorname{Sim}$ & $1(2 \%)$ & $15(22 \%)$ & $0,003^{*}$ \\
\hline & Não & $48(98 \%)$ & $52(78 \%)$ & \\
\hline \multirow[t]{2}{*}{ Prescrição antibiótica } & $\operatorname{Sim}$ & $35(71 \%)$ & $44(66 \%)$ & $0,525^{\#}$ \\
\hline & Não & $14(29 \%)$ & $23(34 \%)$ & \\
\hline \multirow[t]{2}{*}{ Prescrição antibiótica de acordo com NOC } & Sim & $26(72 \%)$ & $28(65 \%)$ & $0,596^{\#}$ \\
\hline & Não & $10(28 \%)$ & $15(35 \%)$ & \\
\hline \multicolumn{5}{|l|}{ OTITE MÉDIA AGUDA } \\
\hline & & $\begin{array}{c}\text { Idade }<2 \text { anos } \\
n=24 \\
n(\%)\end{array}$ & $\begin{array}{c}\text { Idade } \geq 2 \text { anos } \\
n=65 \\
n(\%)\end{array}$ & $p$ \\
\hline \multirow[t]{3}{*}{ Prescrição antibiótica } & Sim & $23(96)$ & $51(78)$ & 0,081 \\
\hline & Não & $1(4)$ & $14(22)$ & \\
\hline & & $\begin{array}{c}\text { Grupo de MGF } \\
n=66 \\
n(\%)\end{array}$ & $\begin{array}{c}\text { Grupo de Pediatria } \\
n=23 \\
n(\%)\end{array}$ & $p$ \\
\hline \multirow[t]{2}{*}{ Atitude expectante } & $\operatorname{Sim}$ & $6(9 \%)$ & $9(39 \%)$ & $0,046^{\#}$ \\
\hline & Não & $60(91 \%)$ & $14(61 \%)$ & \\
\hline \multirow[t]{3}{*}{ Prescrição antibiótica de acordo com NOC } & $\operatorname{Sim}$ & $38(63 \%)$ & $10(71 \%)$ & $0,177^{\#}$ \\
\hline & Não & $22(37 \%)$ & $4(29 \%)$ & \\
\hline & & $\begin{array}{c}1^{\circ} \text { ano } \\
\mathrm{n}=41 \\
\mathrm{n}(\%)\end{array}$ & $\begin{array}{c}2^{\circ} \text { ano } \\
\mathrm{n}=48 \\
\mathrm{n}(\%)\end{array}$ & $p$ \\
\hline \multirow[t]{2}{*}{ Realização de exames complementares de diagnóstico } & Sim & $1(2 \%)$ & $0(0 \%)$ & $0,462^{*}$ \\
\hline & Não & $40(98 \%)$ & $48(100 \%)$ & \\
\hline \multirow[t]{2}{*}{ Prescrição antibiótica } & Sim & $34(82)$ & $41(85)$ & $0,617^{\#}$ \\
\hline & Não & $7(18)$ & $7(15)$ & \\
\hline \multirow[t]{2}{*}{ Prescrição antibiótica de acordo com NOC } & $\operatorname{Sim}$ & $24(71 \%)$ & $28(70 \%)$ & $0,967^{\#}$ \\
\hline & Não & $10(29 \%)$ & $12(30 \%)$ & \\
\hline
\end{tabular}

das queixas, tendo o tratamento sido substituído por antibioterapia com maior espectro de ação. Realizaram esquema da vacina antipneumocócica 65 (73\%) das crianças com este diagnóstico.

\section{Pneumonia adquirida na comunidade}

Nas pneumonias, $42 \%$ realizaram radiografia de tórax para confirmação diagnóstica, mais frequentemente pedida pelo especialista de MGF ( $p=0,009)$ (Tabela 3 ). Não se encontraram diferenças significativas na prescrição antibiótica de doentes com pneumonia entre os profissionais de 
Tabela 5 - Caraterização da escolha de antibioterapia prescrita na amigdalite aguda, otite média aguda, pneumonia adquirida na comunidade e bronquiolite aguda [secção final]

\begin{tabular}{|c|c|c|c|c|}
\hline \multicolumn{5}{|l|}{ PNEUMONIA ADQUIRIDA NA COMUNIDADE } \\
\hline & & $\begin{array}{c}\text { Grupo de MGF } \\
n=25 \\
n(\%)\end{array}$ & $\begin{array}{c}\text { Grupo de Pediatria } \\
n=13 \\
n(\%)\end{array}$ & $p$ \\
\hline \multirow[t]{3}{*}{ Prescrição antibiótica de acordo com NOC } & $\operatorname{Sim}$ & $22(88 \%)$ & $11(85 \%)$ & $0,999^{*}$ \\
\hline & Não & $3(22 \%)$ & $2(15 \%)$ & \\
\hline & & $\begin{array}{l}1^{\circ} \text { ano } \\
n=22 \\
n(\%)\end{array}$ & $\begin{array}{l}2^{\circ} \text { ano } \\
n=16 \\
n(\%)\end{array}$ & $p$ \\
\hline \multirow[t]{2}{*}{ Realização de exames complementares de diagnóstico } & $\operatorname{Sim}$ & $9(41 \%)$ & $7(44 \%)$ & $0,785^{\sharp}$ \\
\hline & Não & $13(59 \%)$ & $9(56 \%)$ & \\
\hline \multirow[t]{2}{*}{ Prescrição antibiótica } & Sim & $19(86)$ & $14(88)$ & $0,773^{\#}$ \\
\hline & Não & $3(14)$ & $2(12)$ & \\
\hline \multirow[t]{2}{*}{ Prescrição antibiótica de acordo com NOC } & Sim & $15(83 \%)$ & $15(100 \%)$ & $0,486^{*}$ \\
\hline & Não & $3(17 \%)$ & $0(0 \%)$ & \\
\hline \multicolumn{5}{|l|}{ BRONQUIOLITE AGUDA } \\
\hline & & $\begin{array}{c}\text { Grupo de MGF } \\
n=31 \\
n(\%)\end{array}$ & $\begin{array}{c}\text { Grupo de Pediatria } \\
n=11 \\
n(\%)\end{array}$ & $p$ \\
\hline \multirow[t]{2}{*}{ Realização de exames complementares de diagnóstico } & Sim & $3(10)$ & $0(\%)$ & $0,531^{\#}$ \\
\hline & Não & $28(90)$ & $11(100 \%)$ & \\
\hline \multirow[t]{3}{*}{ Prescrição antibiótica } & Sim & $7(23)$ & $0(0 \%)$ & $0,296^{\#}$ \\
\hline & Não & $24(77)$ & $11(100 \%)$ & \\
\hline & & $\begin{array}{l}1^{\circ} \text { ano } \\
n=21 \\
n(\%)\end{array}$ & $\begin{array}{l}2^{\circ} \text { ano } \\
\mathrm{n}=21 \\
\mathrm{n}(\%)\end{array}$ & $p$ \\
\hline \multirow[t]{2}{*}{ Realização de exames complementares de diagnóstico } & Sim & $0(0)$ & $3(14)$ & $0,503^{\#}$ \\
\hline & Não & $21(100)$ & $18(86)$ & \\
\hline \multirow[t]{2}{*}{ Prescrição antibiótica } & $\operatorname{Sim}$ & $0(0)$ & $7(33)$ & $0,130^{\ddagger}$ \\
\hline & Não & $21(100)$ & $14(67)$ & \\
\hline
\end{tabular}

*: teste exato de Fisher; \#: teste qui-quadrado de independência; MGF: medicina geral e familiar

saúde (Tabelas 3 e 5), tendo sido prescrito amoxicilina ( $\mathrm{n}$ $=18 ; 55 \%)$, macrólido $(n=12 ; 36 \%)$ e amoxicilina e ácido clavulânico $(n=3 ; 9 \%)$. Nos casos em que foi prescrito um macrólido, $75 \%$ das crianças tinha mais de seis anos. Estavam protegidas com vacina antipneumocócica 27 (71\%) das crianças com este diagnóstico.

\section{Bronquiolite aguda}

Três casos $(7 \%)$ de bronquiolite realizaram radiografia de tórax (Tabela 3). A prescrição antibiótica neste grupo de doentes foi realizada exclusivamente pelo grupo de médicos de MGF e, em dois casos (29\%), o motivo dessa prescrição foi a otite média aguda associada. A escolha de antibioterapia recaiu na amoxicilina e ácido clavulânico ( $\mathrm{n}=$ $4 ; 57 \%)$ e amoxicilina $(n=3 ; 43 \%)$.

\section{Outras patologias respiratórias}

As crianças com infeção respiratória superior inespecífica e laringite aguda foram medicados mais frequentemente com terapêutica sintomática e, em respetivamente $17 \%$ e $30 \%$ dos casos, foi prescrito antibiótico. Este foi realizado em $80 \%$ e $100 \%$ dos casos pelo grupo de MGF. A escolha dessa antibioterapia incluiu macrólido ( $n=21 ; 55 \%)$, amoxicilina $(n=9 ; 24 \%)$, amoxicilina e ácido clavulânico $(n=5$; $13 \%)$ e cefalosporina de segunda geração $(n=3 ; 8 \%)$.

\section{Comparação entre os dois períodos estudados}

A pesquisa na orofaringe do Streptococcus do grupo A nos casos de amigdalite aguda foi mais frequentemente realizada no segundo período estudado $(p=0,003)$, após a apresentação da NOC respetiva. Não se encontraram diferenças estatisticamente significativas entre os dois períodos estudados quanto ao pedido de exames complementares de diagnóstico e escolha de antibiótico de acordo com a NOC em nenhuma das outras patologias (Tabela 5).

\section{Comparação da prescrição antibiótica dependendo das caraterísticas dos doentes}

Não se encontraram diferenças quanto à decisão de prescrever antibioterapia comparando o grupo com e sem comorbilidades $(p=0,462)$. Por outro lado, foi prescrito antibioterapia mais frequentemente aos doentes sem vacina 
Tabela 6 - Relação entre a prescrição antibiótica e comorbilidades associadas (prematuridade, excesso de peso ou obesidade, asma ou sibilância recorrente, problemas do neurodesenvolvimento ou outra) e vacina antipneumocócica

\begin{tabular}{|c|c|c|c|c|}
\hline & & $\begin{array}{l}\text { Saudáveis } \\
n=260 \\
n(\%)\end{array}$ & $\begin{array}{c}\text { Com comorbilidades } \\
n=287 \\
n(\%)\end{array}$ & $p$ \\
\hline \multirow{4}{*}{ Prescrição antibiótica } & Sim & $115(44 \%)$ & $116(40 \%)$ & $0,462^{\#}$ \\
\hline & Não & $145(56 \%)$ & $171(60 \%)$ & \\
\hline & & \multicolumn{3}{|c|}{ Vacina antipneumocócica } \\
\hline & & $\operatorname{Sim}_{n=361}$ & $\begin{array}{c}\text { Não } \\
\mathrm{n}=186 \\
\mathrm{n}(\%)\end{array}$ & $p$ \\
\hline \multirow{2}{*}{ Prescrição antibiótica } & Sim & $128(35 \%)$ & $103(55 \%)$ & $<0,001^{\#}$ \\
\hline & Não & $233(65 \%)$ & $83(45 \%)$ & \\
\hline
\end{tabular}

\#: Teste qui-quadrado de independência

antipneumocócica $(p<0,001)$ (Tabela 6).

\section{DISCUSSÃO}

Os quadros respiratórios infecciosos representaram $35 \%$ do total de consultas por doença aguda, sendo os diagnósticos mais frequentes as infeções respiratórias superiores inespecíficas e amigdalite aguda. Embora tenham representado uma fatia importante dos motivos de consulta aguda no ambulatório, estes resultados são inferiores aos descritos na literatura, segundo a qual mais de 50 a $60 \%$ das consultas que se realizam por doença aguda na idade pediátrica se devem a patologia infecciosa respiratória. . $^{9,36,45}$

A patologia respiratória constitui a causa mais comum de prescrição de antibióticos em idade pediátrica. ${ }^{36,46}$ Os autores desconhecem a existência de estudos em Portugal sobre a frequência de prescrição antibiótica em idade pediátrica, embora tenham havido múltiplas campanhas de sensibilização para a redução desta prescrição na população em geral. Os dados do nosso trabalho indicam que se mantem a necessidade destas campanhas, visto que se pode melhorar a frequência e adequação da antibioterapia.

Prescreveu-se antibiótico em $42 \%$ dos episódios respiratórios infecciosos no período estudado, proporção comparável à apresentada noutros trabalhos (34\% - 48\%). 8,36,47 É amplamente divulgado que a população pediátrica atua como excelente meio de seleção de bactérias resistentes após a utilização de antibióticos dado que esta população contacta frequentemente com os pares da mesma idade, em estabelecimentos de ensino pré-escolar e escolar, favorecendo a transmissão destes agentes patogénicos selecionados. $^{8}$

O tratamento antibiótico frequentemente observado na otite média aguda e amigdalite aguda em crianças, nos diversos estudos, sugerem uma provável sobre prescrição. No caso da amigdalite aguda, a prescrição foi de $68 \%$ (vs $37 \%-93 \%$ ); enquanto que na otite média aguda, foi de $83 \%$ (vs $72 \%$ - 98\%). ${ }^{1,8,47}$ É importante referir que 53\% das crianças com menos de dois anos com amigdalite aguda foram medicadas com antibioterapia, embora neste grupo de idade a etiologia bacteriana seja muito pouco comum. ${ }^{29,48}$ Constatou-se que, após a apresentação da NOC respetiva e da recomendação para a realização de pesquisa na orofaringe do Streptococcus do grupo A, este procedimento tornou-se mais frequente. No entanto, embora a prescrição de antibioterapia tenha sido menor após a apresentação da NOC, não houve diferenças estatisticamente significativas nos dois períodos estudados. Do mesmo modo, a elevada proporção de antibioterapia prescrita, semelhante à observada noutros estudos, não parece ser justificada por se tratarem frequentemente de processos víricos autolimitados os seguintes diagnósticos ${ }^{26}$ : na bronquiolite aguda (17\% vs $11 \%$ - 16\%), ${ }^{1,47}$ infeção respiratória superior inespecífica $(17 \% \text { vs } 6 \%-50 \%)^{1,9}$ e laringite aguda $(30 \%$ vs $3 \%){ }^{1}$ Já nos episódios por pneumonia adquirida na comunidade, prescreveu-se antibiótico em $87 \%$ dos casos (vs 42\%), ${ }^{1}$ tendo sido considerado nos restantes casos uma etiologia vírica. Esta desproporção pode-se dever à provável maior utilização de meios de diagnóstico noutros trabalhos que são realizados em outros contextos em que há maior acesso a estes métodos (radiológico e analítico).

De facto, são antagónicas a necessidade de antibioterapia e o pedido de exames complementares de diagnóstico, porque para minimizar e racionalizar o correto uso da antibioterapia torna-se necessário aumentar o número de pedidos de exames. Isto à custa de iatrogenia com custo-benefício incerto, gerando ansiedade nos cuidadores, pois na maioria dos casos o acesso a esses meios complementares de diagnóstico é moroso.

Constatámos que a cobertura vacinal influenciou a decisão de prescrever a antibioterapia. Por outro lado, as comorbilidades ou patologias prévias não estavam associadas a maior instituição antibiótica nos episódios por patologia respiratória infecciosa.

A adequação dos tratamentos prescritos às recomendações é em geral baixa. Nos casos de otite média aguda em que foi instituída antibioterapia, $72 \%$ dos casos (vs $41 \%)^{8}$ receberam amoxicilina, antibiótico de eleição, excepto nos lactentes com menos de seis meses ou com sintomas graves. ${ }^{28}$ Os episódios por amigdalite aguda em que foi instituída antibioterapia foram tratados com amoxicilina (fármaco de primeira linha juntamente com a penicilina intramuscular) em $70 \%$ dos casos (vs 67\%), ${ }^{8}$ mantendo-se claramente abaixo do idealmente adequado. É de realçar que $16 \%$ receberam amoxicilina com ácido clavulânico, não estando 
indicado nesta patologia, e $10 \%$ dos casos uma cefalosporina de segunda geração, não havendo antecedentes de alergia que o justificassem. ${ }^{29}$ Nos casos de pneumonia adquirida na comunidade, foi mais frequente a prescrição da amoxicilina (55\% vs $70 \%)^{1}$ e macrólido (36\% vs 33\%). ${ }^{1}$ O macrólido foi mais frequentemente utilizado nos doentes em idade escolar, pois corresponde à faixa etária com maior incidência de pneumonias atípicas. ${ }^{49}$

$\mathrm{Na}$ globalidade, a amoxicilina foi o fármaco mais amplamente prescrito. Os macrólidos constituíram, por outro lado, uma franja importante de prescrição (15\%). Como em outros trabalhos (em que a prescrição se situa nos $20 \%$ ), ${ }^{36}$ os médicos continuam a prescrever frequentemente este antibiótico. Foi sugerido que na maioria destas consultas em que este fármaco é prescrito, a antibioterapia não é necessária ou uma alternativa de pequeno espectro poderia ser apropriada. ${ }^{36}$

$\mathrm{Na}$ bibliografia consultada descrevem-se as diferentes causas que motivam um abuso da prescrição antibiótica por parte da equipa médica. Considera-se que esta prescrição na idade pediátrica esteja relacionada com a satisfação dos pais, com o receio de futuras complicações, com as capacidades comunicativas do médico assistente ou da forma como este entende as expectativas dos pais. ${ }^{42,43}$ Perante a realidade descrita, é necessário implementar ações que limitem a prescrição desnecessária e melhorem a adequação destes fármacos. Alguns exemplos seriam a atitude expectante e diferida nas infeções respiratórias ou a utilização de teste rápido de diagnóstico de amigdalite bacteriana quando justificável. ${ }^{28-31,44}$ É necessário promover a formação contínua e consciencialização dos profissionais na sua responsabilidade como prescritores, bem como englobar os pais neste processo, desmistificando mitos das patologias respiratórias e prescrição antibiótica.

Na nossa população, $12 \%$ das crianças e adolescentes tinham recebido pelo menos um ciclo de antibioterapia na unidade de saúde em questão por outro episódio infeccioso no ano transacto. Este valor foi bastante melhor do que o reportado noutros trabalhos $(50 \%-63 \%) .{ }^{47,50}$

A principal limitação deste trabalho é a ausência de diagnósticos microbiológicos que confirmem a etiologia de cada episódio, dificultando o processo de definição da adequação da prescrição. Não obstante, não nos parece provável que justifique o grau de prescrição observada. Para completar o estudo da utilização dos antibióticos será necessário o seguimento do cumprimento da prescrição e da evolução clínica em todos os casos. Este trabalho estudou a associação entre variáveis com base numa análise bivariada, não garantindo que a significância destes testes possa ser mantida numa análise multivariada. ${ }^{51}$

Apesar das possíveis limitações, pode-se destacar a minúcia da informação descrita, onde se engloba o valor do conhecimento em simultâneo do diagnóstico médico e da prescrição realizada.

\section{CONCLUSÃO}

Em geral, este trabalho oferece um bom perfil do número de consultas por causa infecciosa num serviço do ambulatório. O diagnóstico de infeção respiratória superior inespecífica constituiu o principal motivo de doença aguda. Embora a prática clínica em regime ambulatório seja diferente da hospitalar, em que o acesso a meios de diagnóstico com resposta em tempo útil possa influenciar a decisão terapêutica, a prescrição de antibióticos nas infeções respiratórias agudas pediátricas foi elevada e numa proporção significativa a escolha da antibioterapia poderia ser ajustada. Para reduzir o problema das resistências bacterianas no nosso meio, é necessário desenhar e implementar programas de melhoria da qualidade da prescrição a nível dos cuidados de saúde e instituir medidas educacionais à população em geral.

\section{OBSERVAÇÕES}

Trabalho apresentado como comunicação oral no $2^{\circ}$ Congresso Internacional da Criança e Adolescente, que decorreu de 25 a 27 de janeiro de 2018, em Lisboa.

\section{PROTECÇÃO DE PESSOAS E ANIMAIS}

Os autores declaram que os procedimentos seguidos estavam de acordo com os regulamentos estabelecidos pelos responsáveis da Comissão de Investigação Clínica e Ética e de acordo com a Declaração de Helsínquia da Associacção Médica Mundial.

\section{CONFIDENCIALIDADE DOS DADOS}

Os autores declaram ter seguido os protocolos do seu centro de trabalho acerca da publicação de dados.

\section{CONFLITO DE INTERESSES}

Os autores declaram não ter nenhum conflito de interesses na realização do presente artigo.

\section{FONTES DE FINANCIAMENTO}

Os autores declaram não ter recebido subsídios ou bolsas para a elaboração do artigo.

\section{REFERÊNCIAS}

1. Molina CG, Rodríguez-Belvísc MV, Bonet AC, Combelles OV, GarcíaAlgar O. Antibióticos en las infecciones respiratorias en urgencias pediátricas hospitalarias. Arch Bronconeumol. 2014;50:375-8.

2. Baquero F, González J, Martínez D, Olmo V, Orero A, Prieto J. Importancia de la cobertura antimicrobiana y de las resistencias bacterianas en la elección de antibióticos en pediatría. Rev Esp Quimioter. 2009;22:38-47.

3. Goossens H, Ferech M, Vander R, Elseviers M. Outpatient antibiotic use in Europe and association with resistance: a cross-national database

study. Lancet. 2005;365:579-87.

4. Infectious Diseases Society of America and Society for Healthcare Epidemiology of America. Guidelines for developing an institutional program to enhance antimicrobial stewardship. Clin Infect Dis. 2007;44:157-9.

5. Oliva B, Bryant V, Gil M, Timoner J, Álvarez A, Abajo FJ. Prevalencia de uso de antibióticos en la población pediátrica atendida en atención primaria. Estudio en la base de datos BIFAP. Rev Pediatr Aten Primaria. 2009; $11: 17$. 
6. Hernández-Merino A. Uso prudente de antibióticos: propuestas de mejora desde la pediatría comunitaria. Enferm Infecc Microbiol Clin. 2010;28:23-7.

7. Torre M, Pociello N, Rojo P, Saavedra J. Tratamiento antimicrobiano empírico de las infecciones en la infância. [consultado 2018 jun 09]. Disponível em: https://seup.org/pdf_public/pub/tto_antimicrobiano.pdf .

8. Malo S, Bjerrum L, Feja C, Lallana MJ, Poncel A, Rabanaque MJ Prescripción antibiótica en infecciones respiratorias agudas en atención primaria. An Pediatr. 2015;82:412-6.

9. Nash DR, Harman J, Wald ER, Kelleher KJ. Antibiotic prescribing by primary care physicians for children with upper respiratory tract infections. Arch Pediatr Adolesc Med. 2002;156:1114-9.

10. Costelloe C, Metcalfe C, Lovering A, Mant D, Hay AD. Effect of antibiotic prescribing in primary care on antimicrobial resistance in individual patients: systematic review and meta-analysis. BMJ. 2010;340:c2096.

11. Paschke AA, Zaoutis $T$, Conway $\mathrm{PH}$, Xie D, Keren R. Previous antimicrobial exposure is associated with drug-resistant urinary tract infections in children. Pediatrics. 2010;125:664-72.

12. Costelloe $C$, Metcalfe $C$, Lovering A, Mant D, Hay AD. Effect of antibiotic prescribing in primary care on antimicrobial resistance in individual patients: systematic review and meta-analysis. BMJ. 2010;340:c2096.

13. Butler CC, Hillier S, Roberts Z, Dunstan F, Howard A, Palmer S. Antibiotic-resistant infections in primary care are symptomatic for longer and increase workload: outcomes for patients with E coli UTIs. Br J Gen Pract. 2006;56:686-92.

14. Gaur AH, Hare ME, Shorr RI. Provider and practice characteristics associated with antibiotic use in children with presumed viral respiratory tract infections. Pediatrics. 2005;115:635-41.

15. Gonzales R, Malone DC, Maselli JH, Sande MA. Excessive antibiotic use for acute respiratory infections in the United States. Clin Infect Dis. 2001;33:757-62.

16. Grijalva CG, Nuorti JP, Griffin MR. Antibiotic prescription rates for acute respiratory tract infections in US ambulatory settings. JAMA. 2009;302:758-66.

17. Hare ME, Gaur AH, Somes GW, Arnold SR, Shorr RI. Does it really take longer not to prescribe antibiotics for viral respiratory tract infections in children? Ambul Pediatr. 2006;6:152-6.

18. Nash DR, Harman J, Wald ER, Kelleher KJ. Antibiotic prescribing by primary care physicians for children with upper respiratory tract infections. Arch Pediatr Adolesc Med. 2002;156:1114 -9.

19. Nyquist AC, Gonzales R, Steiner JF, Sande MA. Antibiotic prescribing for children with colds, upper respiratory tract infections, and bronchitis. JAMA. 1998;279: 875- 7 .

20. Rossignoli A, Clavenna A, Bonati M. Antibiotic prescription and prevalence rate in the outpatient paediatric population: analysis of surveys published during 2000-2005. Eur J Clin Pharmacol. 2007;63:1099-106.

21. Evidence-based medicine working group. A new approach to teaching the practice of medicine. JAMA. 1992;268:2420-5.

22. National Institute for Health and Clinical Excellence. Clinical guidelines. Prescribing of antibiotics for self-limiting respiratory tract infections in adults and children in primary care. CG69 - Issued: July 2008. [consultado 2018 jun 09]. Disponível em: https://www.nice.org.uk/ guidance/cg69/evidence/full-guideline-196853293.

23. Vallano A, Danes I, Ochoa C. Tratamiento antimicrobiano de las infecciones bronquiales en los servicios de urgencias hospitalarios. An Pediatr. 2004;61:143-9.

24. McCaig LF, Besser RE, Hughes JM. Trends in antimicrobial prescribing rates for children and adolescents. JAMA. 2002;287:3096-102.

25. Patel SJ, Larson EL, Kubin CJ, Saiman L. A review of antimicrobial control strategies in hospitalized and ambulatory pediatric populations. Pediatr Infect Dis J. 2007;26:531-7.

26. Sociedade Portuguesa de Pediatria. Protocolos da Sociedade Portuguesa de Pediatria. [consultado 2018 jun 09]. Disponível em: http://www.spp.pt/conteudos/default.asp?ID=8.

27. Direção Geral de Saúde. Documentos do Dia Europeu dos Antibióticos - Apresentações da sessão científica realizada a 18 de Novembro de 2009. [consultado 2018 jun 09]. Disponível em: https://www.dgs. $\mathrm{pt} / \mathrm{seguranca-do-doente/documentos/dia-europeu-dos-antibioticos-}$ apresentacoes-da-sessao-cientifica-realizada-a-18-de-novembrode-2009.aspx.

28. Direção Geral de Saúde. Diagnóstico e tratamento da otite média aguda em idade pediátrica. Norma de Orientação Clínica da DGS. [consultado 2018 jun 09]. Disponível em: https://www.dgs.pt/directrizes-da-dgs/ normas-e-circulares-normativas/norma-n-0072012-de-16122012.aspx.
29. Direção Geral de Saúde. Diagnóstico e tratamento da amigdalite aguda em idade pediátrica. Norma de Orientação Clínica da DGS. [consultado 2018 jun 09]. Disponível em: https://www.dgs.pt/?cr=23436.

30. Direção Geral de Saúde. Diagnóstico e tratamento da pneumonia adquirida na comunidade em idade pediátrica. Norma de Orientação Clínica da DGS. [consultado 2018 jun 09]. Disponível em: https://www. dgs.pt/directrizes-da-dgs/normas-e-circulares-normativas/norma-n0192012-de-26122012.aspx.

31. Direção Geral de Saúde. Diagnóstico e tratamento da bronquiolite aguda na idade pediátrica. Norma de Orientação Clínica da DGS. [consultado 2018 jun 09]. Disponível em: https://www.dgs.pt/directrizes-da-dgs/ normas-e-circulares-normativas/norma-n-0162012-de-19122012.aspx.

32. Direccion General de Aseguramiento y Planificacion Sanitaria. Agencia de Evaluacion de Tecnologias Sanitarias. Ministerio de Sanidad y Consumo. Informe sobre resistencia microbiana: ¿que' hacer? Med Clin. 1995;106:267-79.

33. Palop V, Melchor A. Reflexiones sobre la utilizacion de antibioticos en atencion primaria. Aten Primaria. 2003;32:42-7.

34. Direção Geral de Saúde. Programa de Prevenção e Controlo de Infeções e de Resistência aos Antimicrobianos - Relatório 2017. [consultado 2018 jun 09]. Disponível em: https://www.dgs.pt/documentos-e-publicacoes/ programa-de-prevencao-e-controlo-de-infecoes-e-de-resistencia-aosantimicrobianos-relatorio-2017.aspx.

35. Macfarlane J, Holmes W, Macfarlane R, Britten N. Influence of patients expectations on antibiotic management of acute lower respiratory tract illness in general practice: questionnaire study. BMJ. 1997;315:1211-4.

36. Hersh AL, Shapiro DJ, Pavia AT, Shah SS. Antibiotic prescribing in ambulatory pediatrics in the United States. Pediatrics. 2011;128:105361.

37. Brooks L, Shaw A, Sharp D, Hay AD. Towards a better understanding of patients' perspectives of antibiotic resistance and MRSA: a qualitative study. Fam Pract. 2008;25:341-8.

38. Butler CC, Rollnick S, Pill R, Maggs-Rapport F, Stott N. Understanding the culture of prescribing: qualitative study of general practitioners' and patients' perceptions of antibiotics for sore throats. BMJ. 1998;317:63742.

39. Kumar S, Little P, Britten N. Why do general practitioners prescribe antibiotics for sore throat? Grounded theory interview study. BMJ. 2003;326:138.

40. De Jong J, Bos JH, Vries TW, Berg LT. Antibiotic use in children and the use of medicines by parents. Arch Dis Child. 2012;97:578-81.

41. Mangione-Smith R, Elliott MN, Stivers T, McDonald L, Heritage J, McGlynn EA. Racial/ethnic variation in parent expectations for antibiotics: implications for public health campaigns. Pediatrics. 2004;113:e385-94.

42. Andre M, Hedin K, Hakansson A, Molstad S, Rodhe N, Petersson C More physician consultations and antibiotic prescriptions in families with high concern about infectious illness-adequate response to infectionprone child or self-fulfilling prophecy? Fam Pract. 2007;24:302-7.

43. Vazquez-Lago JM, Lopez-Vazquez P, Lopez-Duran A, Taracido-Trunk $M$, Figueiras A. Attitudes of primary care physicians to the prescribing of antibiotics and antimicrobial resistance: a qualitative study from Spain. Fam Pract. 2012;29:352-60.

44. Hernandez-Merino A. Uso prudente de antibióticos: propuestas de mejora desde la pediatría comunitaria. Enferm Infec Microbiol Clin. 2010;28:23-7

45. Gonzalez J, Ochoa C, Alvarez G. Manejo racional de la antibioterapia en infecciones otorrinolaringológicas en la infancia: revisión crítica de las mejores pruebas científicas. Acta Otorrinolaringol Esp. 2006;57:66-81.

46. Raz R, Hassin D, Kitzes-Cohen R, Rottensterich E. Antibiotic prescribing for adults and children in Israeli emergency rooms. Int J Antimicrob Agents. 2003;22:100-5.

47. Rey CC, Ballesteros RA, Méndez MY, Domínguez AO. Patrones de prescripción de antibióticos en atención primaria. ¿Usamos racionalmente los antibióticos en pediatría? An Esp Pediatr. 2000;52:157-63.

48. Piñeiro R, Hijano F, Álvez F, Fernández A, Silva JC, Pérez C, et al. Documento de consenso sobre el diagnóstico y tratamiento de la faringoamigdalitis aguda. An Pediatr. 2011;75:342. e1-13.

49. McIntosh K. Community-acquired pneumonia in children. N Engl J Med. 2002;346:429-37.

50. Thrane N, Steffensen FH, Mortensen JT, Schonheyder HC, Sci DM, Sorensen HT. A population-based study of antibiotic prescriptions for Danish children. Pediatr Infect Dis J. 1999;18:333-7.

51. Agresti A. Categorical data analysis. $2^{\text {nd }}$ ed. New York: John Wiley \& Sons; 2002. 